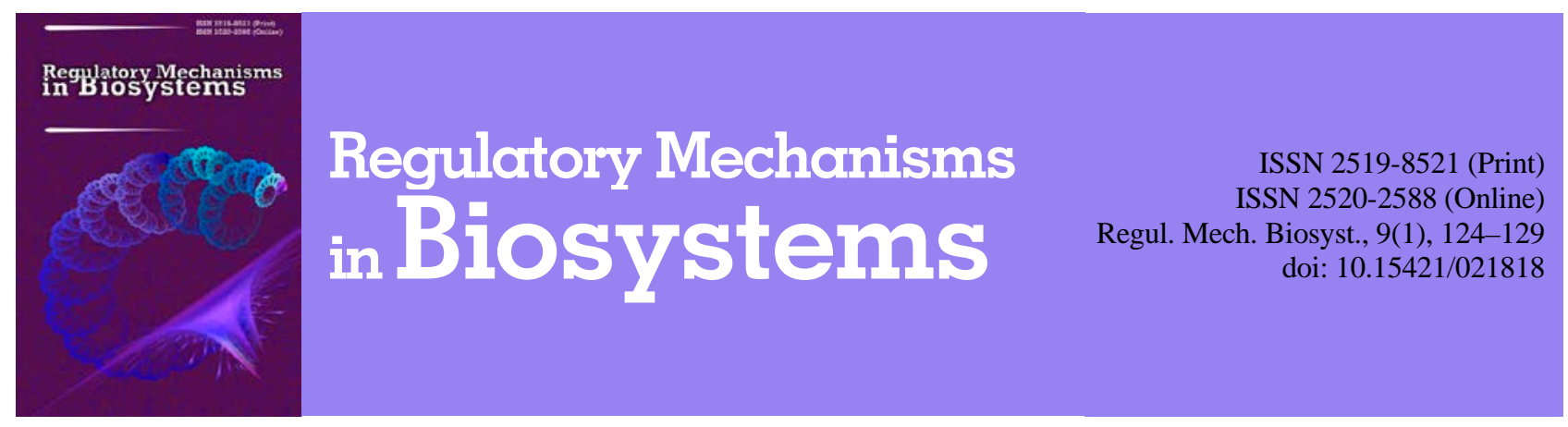

\title{
Violations of cell-molecular mechanisms of bone remodeling under influence of glucocorticoids
}

\author{
S. B. Pavlov, N. M. Babenko, M. V. Kumetchko, O. B. Litvinova \\ Kharkiv Medical Academy of Postgraduate Education, Kharkiv, Ukraine
}

Article info

Received 07.02.2018

Received in revised form 26.02.2018

Accepted 28.02.2018

Kharkiv Medical Academy of Postgraduate Education, Amosova st., 58,

Kharkiv, 61176, Ukraine. Tel. + 38-057-711-80-48.

E-mail: cndl@med.edu.ua

\begin{abstract}
Pavlov, S. B., Babenko, N. M., Kumetchko, M. V., \& Litvinova, O. B. (2018). Violations of cell-molecular mechanisms of bone remodeling under influence of glucocorticoids. Regulatory Mechanisms in Biosystems, 9(1), 124-129. doi: $10.15421 / 021818$
\end{abstract}

The fact is disturbance of the processes of bone tissue remodeling leads to a change in the balance between synthesis and resorption of bone and the development of osteoporosis. The most common cause of secondary osteoporosis is the use of glucocorticoid therapy. The aim of this study is to investigate the cellular-molecular mechanisms of disturbance of the processes of bone remodeling regulation, reflected by hormones and intercellular mediators (for example parathyroid hormone, calcitonin, RANKL, osteoprotegerin, P-selectin, interleukin- 17 , transforming growth factor- $\beta 1$, adiponectin and visfatin) on the background experimental glucocorticoid osteoporosis. The experimental study carried out in two groups of white female rats. Disturbance of bone tissue remodeling was verified by histological examination of the femoral head, vertebrae of the thoracic and lumbar spine of rats and the measurement of bone density. The study of the levels of hormones and intercellular mediators in the blood serum of animals was carried out by the method of enzyme immunoassay. The bone mineral density of the experimental group was reduced compared to the bone mineral density of the control group. The study of the histostructure of the femoral head and vertebrae in rats of the experimental group in comparison with the animals of the control group revealed changes in the structural organization of bone tissue, confirmed by histomorphometry, indicating inhibition of the processes of osteosynthesis. The article analyzes the nature of the involvement of hormones and cytokines in pathogenetic mechanisms of development of bone tissue disorders. The levels of cytokines RANKL, osteoprotegerin, interleukin-17 and calcitonin in the blood serum of animals of the group with the violation of bone tissue remodeling by glucocorticoids were higher than in intact animals. Serum levels of P-selectin, parathyroid hormone, transforming growth factor- $\beta 1$, adiponectin and visfatin were lower than similar levels in animals from the control group. The use of glucocorticoids increases the expression of RANKL and inhibits the synthesis of osteoprotegerin, resulting in stimulation of bone resorption. The effect of glucocorticoids in the experimental model is realized by changing the production of the studied hormones, cytokines and adhesion molecules. These changes stimulate the apoptosis of osteoblasts and inhibit their proliferation and differentiation, which is another mechanism of bone loss. Correlations found during the study reflect the relationship in the system of regulation of bone tissue remodeling under the influence of glucocorticoids. A complex system for regulating bone remodeling, which includes many regulatory pathways and their interactions, requires further study.

Keywords: cytokines; hormones; glucocorticoids; remodeling of a bone tissue; adipokines

\section{Introduction}

When bone remodeling processes are disturbed, the balance between formation and resorption of bone tissue changes, controlled and coordinated by different types of bone cells. This often leads to the development and progression of osteoporosis, which, according to World Health Organization data, is the second most important health problem after cardiovascular disease (Klimova et al., 2014). Secondary osteoporosis refers to bone disorders, which are a secondary complication of various diseases. The most common cause of secondary osteoporosis is the consequences of glucocorticoid therapy. Glucocorticoids reduce bone density through several mechanisms: suppression of sex steroid hormones, inhibition of gastrointestinal absorption and renal calcium reabsorption, stimulation of parathyroid hormone secretion and inhibition of bone formation resulting from a change in the balance between osteoclasts and osteoblast activity (Kaneko et al., 2012). Glucocorticoids are capable of altering the proliferation and metabolism of bone cells (Brennan-Speranza et al., 2012). It is assumed that the development of glucocorticoid-induced osteoporosis occurs in two steps. In the initial, rapid steps of the use of glucocorticoids, bone loss occurs due to both a reduction in bone formation, and as a result of accelerated bone resorption. In the second, slower phase, the rate of osteoclast-mediated bone resorption slows down; the predominance is the suppression of bone formation (Canalis et al., 2007). Thus, with prolonged glucocorticoid therapy, the number of osteoclasts is usually maintained in the normal range, while the number of osteoblasts is significantly reduced, in contrast to postmenopausal osteoporosis, in which there is increased bone resorption (Weinstein, 2011). Apoptosis of osteoblasts, induced by the constant use of glucocorticoids, is identified as the main cause of osteoporosis, bone loss and fractures.

The process of bone remodeling is controlled by various local and systemic factors. Parathyroid hormone and calcitonin are some of the major hormonal regulators of bone resorption.

Parathyroid hormone is synthesized by parathyroid glands. Its main function is to maintain blood calcium homeostasis. The effect of parathyroid hormone is an increase in the concentration of calcium in the blood, a decrease in the calcium content in the bones (demineralization of the bone matrix) and a decrease in the phosphate content in the blood 
plasma. Excess content of parathyroid hormone leads to a disorder of bone tissue, endocrine disease such as hyperparathyroidism, bone disease such as osteoporosis.

Calcitonin, which is a 32-amino acid hormone, is secreted by the cells of the thyroid gland. This hormone mainly acts, supplementing the function of parathyroid hormone, counteracting increased bone resorption caused by parathyroid hormone.

In addition to systemic hormonal regulation, a number of cytokines and growth factors are involved in bone remodeling. At the molecular level, bone resorption is regulated through the interaction of the receptor activator of nuclear factor- $\mathrm{kB}$ ligand (RANKL) and osteoprotegerin (OPG) against the background of the permissive action of the macrophage colony-stimulating factor. RANKL functions as a key factor for osteoclastogenesis, since its binding to the RANK receptor (receptor activator of nuclear factor- $\kappa \mathrm{B}$ ) promotes the activation of osteoclasts and the resorption of bone tissue. Osteoprotegerin reduces RANKLRANK interactions and thereby inhibits osteoclastogenesis (RemuzgoMartínez et al., 2016). In this regard, the balance between RANKL and osteoprotegerin actually determines the amount of resorpted bone and the degree of change in bone mineral density.

It has been established that the RANKL/RANK/OPG-cytokine system that initiates osteoblasto- and osteoclastogenesis in bone tissue induces differentiation of osteoblasts and osteoclasts, as well as the process of mineralization of the vessel walls (Sage et al., 2010). RANKL and osteoprotegerin can be the molecular link between calcification of the arteries and bone resorption, which underlies the clinical combination of vascular disease and osteoporosis. In the regulation of bone remodeling, adipokines are also involved, affecting bone remodeling by suppressing intracellular osteogenic signals, while simultaneously promoting the secretion of adipogenic signaling molecules such as adiponectin and visfatin (Muruganandan \& Sinal, 2014).

The aim of the study was to investigate the cellular-molecular mechanisms of disturbance of bone tissue remodeling regulation processes, as reflected by intercellular mediators (for example parathyroid hormone, calcitonin, RANKL, osteoprotegerin, P-selectin, interleukin-17, transforming growth factor- $\beta 1$, adiponectin and visfatin) in experimental glucocorticoid osteoporosis.

\section{Materials and methods}

The experimental study was carried out in two groups of white female rats at the age of 9 months with a mass of $250 \pm 30$ g. First group is a group of animals with a violation of bone tissue remodeling caused by glucocorticoids - 18 rats, second group (control) - 10 rats. Creation of a model of experimental disturbance of bone tissue remodeling by glucocorticoids was carried out by administration of dexamethasone phosphate $6 \mathrm{mg} / \mathrm{kg}$ by weight intramuscularly twice a week for a month (Liu et al., 2011). Control group - intact animals.

The experiments were carried out in accordance with the principles of the "European Convention for the Protection of Vertebrate Animals used for Experimental and Other Scientific Purposes” (Strasbourg, 1986) and the "General ethical principles of animal experimentation" approved by the First National Congress on Bioethics (Kiev, 2001).

Disturbance of bone remodeling was monitored by direct measurement of bone density, which was calculated as the ratio of bone mass (grams) to its volume (centimeters cubic) (Podkovkin et al., 2008).

For histological examination, the femoral head, as well as the vertebrae of the thoracic and lumbar spine of the rats, were isolated. The material was fixed in $10 \%$ neutral formalin, decalcified in 5\% nitric acid, encased in paraffin according to a conventional technique (Sarkisov \& Perov, 1996). Sections 7-10 $\mu \mathrm{m}$ thick were stained with hematoxylin and eosin,van Gieson's picrofuchsin , analyzed in the field of view of the "Primo Star" microscope (Carl Zeiss). Photomicrographs of the preparations were performed using a Microocular digital camera.

Morphometric analysis of histopreparations was carried out using a light microscope LOMO (lens ${ }^{\times} 10$, eyepiece ${ }^{\times} 8$ ). The central sections were analyzed, in each individual case (at least three). In the area of the femoral head of animals with experimental osteoporosis, the severity of osteopenic manifestations was assessed for the following parameters: width of the cortex and thickness of the bone trabeculae (estimated with the help of an ocular screw micrometer MOV-1-16 ${ }^{\times}$), and also by the ratio of the volume of bone trabeculae to the total volume of spongy bones (conducted using the ocular-mesh insert G. G. Avtandilov (289 points of intersection)), guided by the method proposed by the author (Avtandilov, 1990). As a control, similar indices of intact animals were used.

The studies were carried out in the blood serum of animals by the method of enzyme immunoassay. The levels of parathyroid hormone, calcitonin and transforming growth factor- $\beta 1$ were determined using DRG kits (Germany). To quantify the levels of RANKL, a set of reagents “ampli-sRANKL" Biomedica (Austria) was used. The levels of osteoprotegerin were determined using the Human Osteoprotegerin Instant eBioscience kit (Austria). P-selectin levels were determined using the Human sP-selectin Platinum ELISA kit eBioscience (Austria). A set of Vector-Best (Russia, Novosibirsk) was used to quantify interleukin-17 levels. Visfatin levels were determined using the RayBio kit (USA), adiponectin levels, using the BioVendor kit (Czech Republic).

The statistical processing of the results was carried out using the Statistica 6.0 analysis package using the Kruskal-Wallis non-parametric ANOVA criterion for independent samples and correlation analysis. The results are presented in the form $x \pm S E$, where $x$ is the arithmetic mean, SE is the standard error of the arithmetic mean. Statistically significant differences were considered at $\mathrm{P}<0.05$.

\section{Results}

The measured bone mineral density of the animal group with the violation of bone tissue remodeling with glucocorticoids was significantly decreased in comparison with the bone mineral density of the animals in the control group $\left(1.37 \pm 0.041\right.$ and $1.62 \pm 0.059 \mathrm{~g} / \mathrm{cm}^{3}$ respectively, $\mathrm{P}<0.05)$. A review of the histological specimens of the vertebral bodies and the proximal femur in the rats of the control group showed a typical structure of bone tissue. The spongy bone was represented by wide anastomosing bone trabeculae, separated by intertrabecular spaces that contained red bone marrow. In the thickness of the beams, lacunae with osteocyte bodies were uniformly distributed; dark blue, slightly wavy cement lines were clearly contoured. The cortical layer, represented by a compact bone, was of sufficient width all along.

A study of the histostructure of the head of the femur and vertebrae of rats with experimental osteoporosis in comparison with the animals of the control group revealed changes in the structural organization of bone tissue. In the proximal part of the femur and the bodies of the vertebrae of the thoracic and lumbar regions of experimental animals, similar changes were found, which testify to the inhibition of bone formation processes.

In the spongy substance of bone tissue, these changes were associated with the thinning of the trabeculae and the dilution of the trabecular network. There was a decrease in the number of trabeculae and their contacts between themselves and the cortex (Fig. 1). Most of the trabeculae were thinned, had uneven edges and blind ends, which indicates the prevalence of bone resorption processes. Uneven staining of the matrix of bone tissue, uneven distribution of osteocytes, basophilia, thickening of cement lines in the areas was observed. Microcracks of trabeculae along the stratification of cement lines and single microfractures were noted (Fig. 2).

Cortex on the parts of the head of the femur and vertebral bodies was thinned and uneven in width. We detected expanded lacunae of osteocytes, vascular channels and single cavities filled with red bone marrow and reticulo-fibrous tissue, which reflects the process of rarity of compact bone. Osteocytes were of various sizes, their distribution in the regions was uneven. As a manifestation of a violation of calcification, sharply basophilic walls of a part of lacunae were revealed.

The results obtained are confirmed by histomorphometry - the method of objective evaluation of the state of bone tissue remodeling at the cellular and tissue levels. Morphometric examination of histopreparations of the femoral head recorded quantitative and qualitative changes in the parameters of bone tissue. It was found that the ratio of the volume of bone trabeculae to the volume of the spongy bone in the examined 
area in animals with experimental osteoporosis was reduced by $32.9 \%$, the width of the cortical layer and the thickness of the trabeculae were less (by 56.8\% and 56.1\% respectively, $\mathrm{P}<0.05$ ) in comparison with the parameters of the animals of the control group, which indicates a decrease in bone formation.

Thus, histological analysis and reduction of bone mineral density showed that the use of dexamethasone enabled us to obtain a model of bone tissue remodeling disturbance.
The levels of cytokines RANKL, osteoprotegerin, interleukin-17 and calcitonin in the blood serum of animals of the group with the disorder of bone tissue remodeling by glucocorticoids were higher than in intact animals, but the difference was statistically insignificant (Table 1). The most significant was an increase in the concentration of osteoprotegerin (by 55.5\%) and RANKL (by 35.9\%) in the serum of animals with experimental osteoporosis, which corresponds to the contribution of these cytokines to bone remodeling.
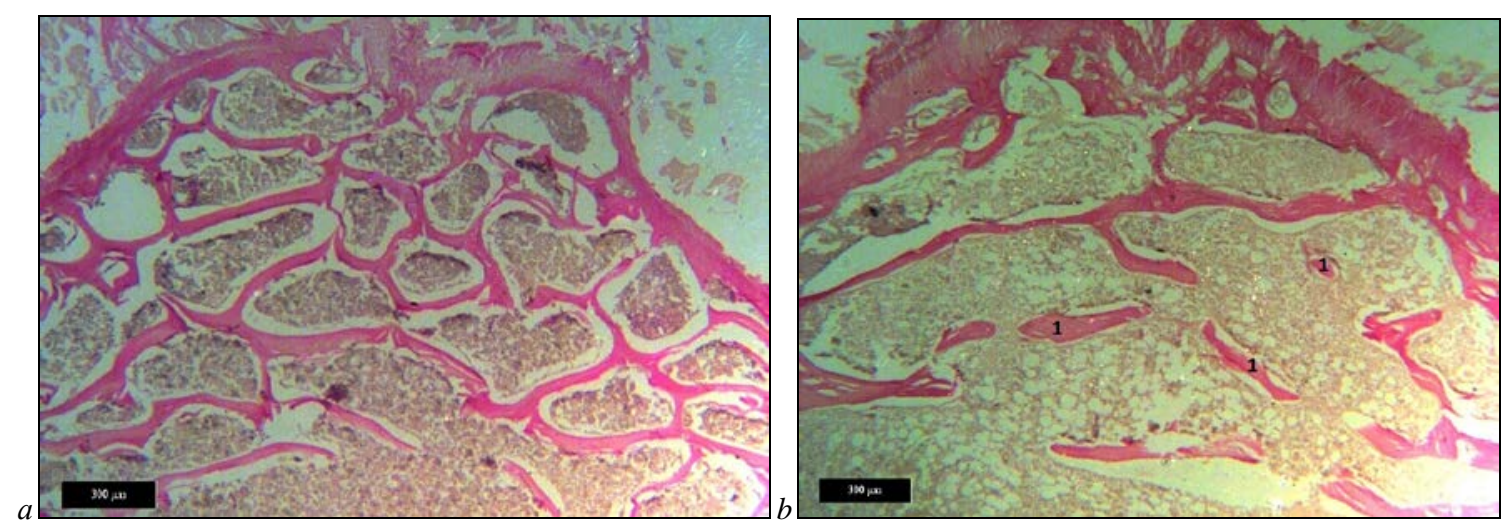

Fig. 1. Areas of the vertebrae of the lumbar spine of the rats:

$a$-the control group, $b$ - with experimental osteoporosis; 1 - isolated trabeculae; van Gieson's picrofuchsin
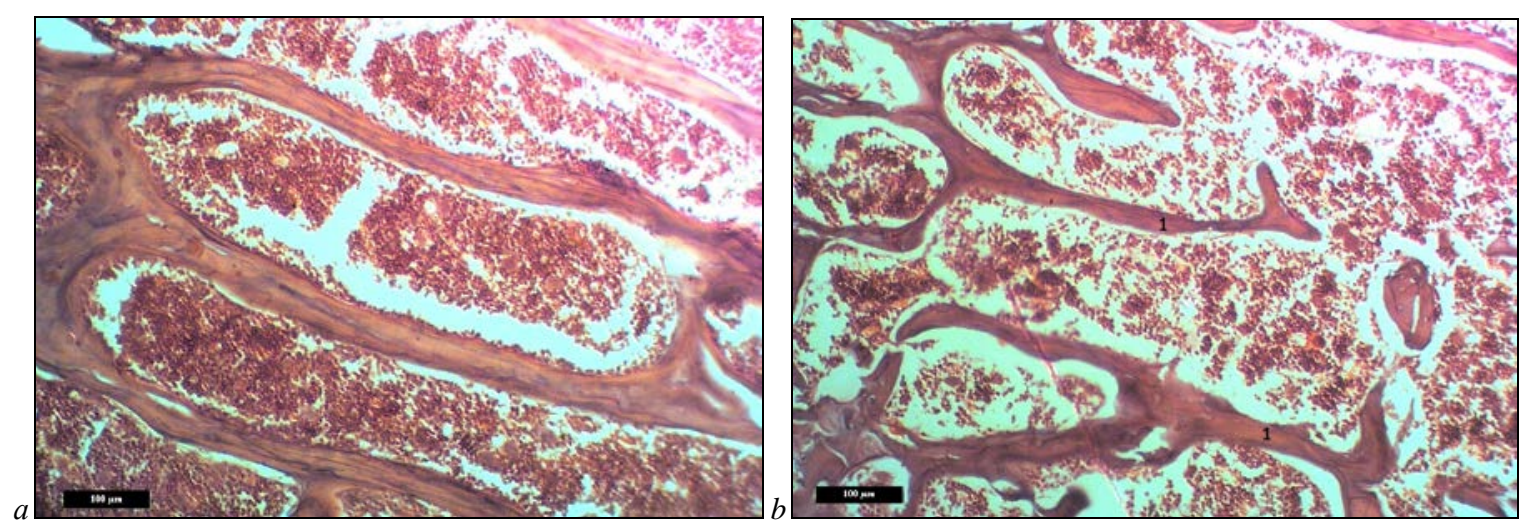

Fig. 2. Areas of the vertebrae of the thoracic spine of rats:

$a$ - the control group, $b$ - with experimental osteoporosis; 1 - thinned trabeculae, not forming a network; hematoxylin-eosin

Table 1

Levels of cytokines in the control and experimental groups $(\mathrm{x} \pm \mathrm{SE})$

\begin{tabular}{lcc}
\hline & \multicolumn{2}{c}{ Groups } \\
\cline { 2 - 3 } \multicolumn{1}{c}{ Cytokine } & $\begin{array}{c}\text { control, } \\
\mathrm{n}=10\end{array}$ & $\begin{array}{c}\text { group with the violation of bone } \\
\text { tissue remodeling by } \\
\text { glucocorticoids, } \mathrm{n}=18\end{array}$ \\
\hline $\begin{array}{l}\text { Receptor activator of nuclear } \\
\text { factor- } \mathrm{\kappa B} \text { ligand, pmol/ }\end{array}$ & $0.131 \pm 0.021$ & $0.178 \pm 0.046$ \\
Osteoprotegerin, pg/ml & $22.7 \pm 2.2$ & $35.3 \pm 9.5$ \\
P-selectin, $\mathrm{ng} / \mathrm{ml}$ & $2.23 \pm 0.09$ & $1.66 \pm 0.07 *$ \\
Interleukin-17, pg/ml & $28.6 \pm 1.3$ & $29.0 \pm 1.3$ \\
Transforming growth & $26.5 \pm 1.6$ & $26.2 \pm 1.5$ \\
factor- $\beta_{1}, \mathrm{ng} / \mathrm{ml}$ & $0.663 \pm 0.021$ & $0.631 \pm 0.014$ \\
Adiponectin, $\mu \mathrm{g} / \mathrm{ml}$ & $141.6 \pm 26.7$ & $133.3 \pm 22.9$ \\
Visfatin, $\mathrm{ng} / \mathrm{ml}$ & $7.85 \pm 1.03$ & $5.24 \pm 0.95$ \\
Parathyroid hormone, pg/ml & $8.81 \pm 0.85$ & $9.52 \pm 1.37$ \\
Calcitonin, $\mathrm{pg} / \mathrm{ml}$ &
\end{tabular}

Note: ${ }^{*}-\mathrm{P}<0.05$ compared with the control group.

At the same time, in the group of animals with disturbed bone remodeling of glucocorticoids, the serum P-selectin level was signifycantly lower (by 25.8\%) in the same level in animals from the control group $(\mathrm{P}<0.05)$. The concentration of parathyroid hormone decreased by 33.2\% ( $\mathrm{P}>0.05$ ), while the decrease in the levels of transforming growth factor- $\beta_{1}$, adiponectin and visfatin in the serum of animals in the experimental group was insignificant and statistically insignificant.
Research on intra group interrelations between indicators of cytokines revealed several correlations of different force In the control group, the presence of an inverse strong bond was established $(r=-$ $0.899, \mathrm{P}<0.05$ ) between the content of visfatin and interleukin-17, medium strength feedback $(\mathrm{r}=-0.695, \mathrm{P}<0.05)$ between RANKL and transforming growth factor- $\beta_{1}$. In a group with experimental osteoporosis, these correlations became insignificant $(\mathrm{r}=0.133$ and $\mathrm{r}=$ -0.006 respectively, $\mathrm{P}>0.05$ ). In a group with a disturbance of bone tissue remodeling by glucocorticoids, a direct relationship of average force $(r=0.626, P<0.05)$ between the levels of visfatin and P-selectin was revealed. In the control group, a similar relationship was not found $(\mathrm{r}=-0.056, \mathrm{P}>0.05)$. The presence of a connection between the studied parameters is explained by their mutual influence on the metabolism of bone tissue.

\section{Discussion}

The observed increase in serum RANKL level in animals in the experimental group may contribute to the loss of bone mass through the RANK/RANKL/OPG pathway, which corresponds to a decrease in bone mineral density in these animals. Binding of osteoprotegerin to RANKL prevents the destruction of bone tissue. The balance between the formation and destruction of bone determines the level of activation of osteoclasts (Luan et al., 2012). The administration of high doses of dexamethasone to rats inhibits the differentiation of osteoblasts and cell 
proliferation. The use of glucocorticoids increases the expression of RANKL and inhibits the synthesis of osteoprotegerin, resulting in stimulation of bone resorption. In our study there is an increase in the concentration of osteoprotegerin in the blood serum of animals with experimental disturbance of bone tissue remodeling induced by glucocorticoids, which can be considered as a compensatory reaction to glucocorticoid-induced bone resorption. Osteoprotegerin is an important prognostic factor; in addition to regulating bone turnover, this cytokine inhibits vascular calcification and modulates inflammation in the vascular wall (Scialla et al., 2011). Osteoprotegerin is stimulated by numerous inflammatory mediators, such as interleukin- 1 , tumor necrosis factor- $\alpha$, transforming growth factor- $\beta$ and interferon- $\gamma$. Osteoprotegerin stimulates the expression of adhesion molecules and leukocyte infiltration in the walls of the vessels, which promotes the expression of RANKL.

In our study, there was a slight increase in the concentration of proinflammatory interleukin-17, produced by activated T-cells. Interleukin17 supports osteoclastogenesis, depending on the signal path of RANKL-RANK. Cytokine increases the sensitivity of osteoclast precursors to RANKL by increasing RANK expression on osteoclastic progenitors (Adamopoulos et al., 2010). Moreover, interleukin-17 activates the expression of RANKL in osteoblasts, synovial and mesenchymal cells, thereby increasing the RANKL/OPG ratio and enhancing osteoclastogenesis. Thus, interleukin-17 through osteoblasts indirectly affects bone resorption, which ultimately contributes to the loss of bone tissue, reflected by a decrease in bone mineral density in our study. At the same time, interleukin-17 is an important mediator of the response to the treatment of glucocorticoids. According to the literature, dexamethasone reduces the expression of interleukin-17A in models of asthma in mice (Lu et al., 2013), or does not affect the production of interleukin-17 (Zeng et al., 2015), or Th17 cells (T-helpers 17) show limited sensitivity to dexamethasone (McKinley et al., 2008). Thus, the level of this intercellular mediator is apparently due to its ability to amplify the differentiation of osteoclasts and their functional activity by means of RANKL (direct and indirect pathway), and due to the effect of glucocorticoids on the T-cells producing this cytokine.

Suppression of the formation of bone tissue occurs as a result of the physiological effect of parathyroid hormone through the effect on the population of osteoblasts that secrete insulin-like growth factor 1 and cytokines that stimulate the metabolism of osteoclasts. Activated osteoclasts secrete alkaline phosphatase and collagenase, which leads to bone resorption. Reduction in the concentration of parathyroid hormone in our study, apparently, can be explained by a violation of the balance between the opposite effects that arise with the use of glucocorticoids. Glucocorticoids inhibit calcium absorption in the intestine and increase urinary calcium excretion (by inhibiting renal tubular reabsorption of calcium). This, as a rule, leads to a decrease in the levels of ionized calcium in the serum, which in turn can lead to an increase in the levels of parathyroid hormone. At the same time, an increase in bone resorption has an opposite effect on the secretion of parathyroid hormone by parathyroid glands, leading to a decrease in the levels of this hormone (Mazziotti et al., 2016).

In turn, calcitonin, being an antagonist of parathyroid hormone, reduces the concentration of calcium in the serum and slows the activity of osteoclasts, reducing the destruction of bone. The mechanisms of the effect of calcitonin on remodeling of bone tissue remain unclear until the end. Calcitonin has been shown to modify cell cultures of osteoblasts and osteocytes (Plotkin et al., 1999). The hypothesis based on the finding of calcitonin receptors on osteocytes is that calcitonin can potentially modify osteocyte products, such as fibroblast growth factor 23 or, more importantly, sclerostin, known as bone growth regulator (Davey \& Findlay, 2013). Changes in the level of the hormone in the experimental group, apparently, are due to the effect of dexamethasone and are compensatory in nature, aimed at maintaining homeostasis, in particular, the level of ionized calcium.

Glucocorticoids also regulate many aspects of endothelial physiology, including the expression of adhesion molecules, the production of proinflammatory cytokines, and the maintenance of endothelial barrier integrity. The inhibitory effect of dexamethasone on the expression of P-selectin (Xiping et al., 2010), described as a biomarker of the develop- ment of atherosclerosis, is described. P-selectin is an adhesive transmembrane glycoprotein that is transferred to the cell surface from platelet $\alpha$-granules and Weibel-Palade bodies of endothelial cells during activation of these cells, while the protein is partially released into the blood plasma, where it circulates in a soluble form. The binding of P-selectin to a specific glycoprotein ligand-1 allows interaction between leukocytes and endothelial cells, leukocytes and platelets, platelets and endothelium, thus involving cells in the emerging thrombus (Zubairova et al., 2013). Reduction of the concentration of P-selectin in the blood serum of animals of the group with experimental disturbance of bone tissue remodeling by glucocorticoids may be explained by the inhibitory effect of dexamethasone.

During the bone remodeling cycle, direct and indirect communications between bone cells are performed in a process called a binding mechanism. Transforming growth factor- $\beta 1$, along with factors such as insulin-like growth factor, bone morphogenetic proteins, fibroblast growth factor, and platelet-derived growth factor appear to act as a coupling factor because it is stored in bone matrix and is released when bone resorption (Florencio-Silva et al., 2015). This cytokine is a growth factor that is involved in the control of proliferation, migration, differrentiation and survival of many cell types. Transforming growth factor$\beta_{1}$ can stimulate the proliferation of osteoblasts and regulate the functions of osteoclasts, thus being a regulator of bone remodeling. It affects the formation and resorption of bone and the production of some proinflammatory cytokines. This cytokine is an important modulator of vascular remodeling in atherosclerosis (Wan et al., 2012). Although transforming growth factor- $\beta_{1}$ enhances proliferation and early differrentiation of osteoblasts, blocks their apoptosis, promoting the formation of bone tissue, this cytokine is able to inhibit osteoclastogenesis by activating the transcription factor Smad4 in osteoclasts (Morita et al., 2016). Transforming growth factor- $\beta_{1}$ has a double effect on osteoblasts: lower levels of this cytokine contribute to the differentiation of osteoclasts, while higher concentrations inhibit it (by increasing osteoprotegerin expression that inhibits RANKL-induced osteoclast differentiation) (Crane et al., 2016). These data confirm the correlation between the levels of RANKL and transforming growth factor- $\beta_{1}$ revealed by us. Glucocorticoids stimulate apoptosis of osteoblasts, inhibit their proliferation and differentiation depending on the dose, duration and stage of cellular differentiation (Yamashita et al., 2014). Apparently, the level of transforming growth factor- $\beta_{1}$ in the blood serum of animals of the group with the experimental violation of bone tissue remodeling by glucocorticoids is due both to its dysregulatory role in the stages of osteoblast and osteoclast differentiation and to the action of dexamethasone.

Regulation of bone remodeling includes not only osteoblastic and osteoclastic cell lines, but also other bone marrow cells. Adipocytes are obtained from the same precursor cells as osteoblasts, mesenchymal stem cells, and the balance between the two types of cells is important for bone remodeling. The number of adipocytes in the bone marrow varies in different pathophysiological conditions. Elevated levels of glucocorticoids also contribute to adipogenesis, limiting osteoblastogenesis ( $\mathrm{Li}$ et al., 2013). The dexamethosone used in our experimental study serves as a potent adipogenic factor. Accelerated adipogenesis in the bone marrow is associated with the progression of osteoporosis (Sharma et al., 2014).

Adiponectin is the most common adipokine, the protein mediator, first described as a fatty tissue product secreted by adipocytes and regulating energy metabolism. Adiponectin plays a key role in various processes, such as energy metabolism, inflammation and cell proliferation, showing insulin-sensitive, anti-inflammatory, anti-atherosclerotic and antidiabetic properties (Padmalayam \& Suto, 2013). It inhibits the production of adhesion molecules in endothelial cells. Adiponectin and its receptors are expressed in bone tissues and participate in bone metabolism. However, the literature presents contradictory results on the role of adiponectin in remodeling bone tissue. Adiponectin affects the differentiation of mesenchymal stem cells into preosteoblasts, as well as the proliferation and maturation of osteoblasts, which contributes to the regeneration of bone (Liu et al., 2013). In addition to positive modulation of osteoblasts, adiponectin also has a negative effect on os- 
teoclasts. It increases the apoptosis of osteoclasts and reduces the proliferation of osteoclast precursor cells (Tu et al., 2011). Adiponectin inhibits the osteoclastic differentiation induced by macrophage colony-stimulating factor and RANKL, both mouse macrophages and human $\mathrm{CD}_{14}+$ mononuclear cells and, consequently, inhibits bone resorption activity of osteoclasts. It is reported that in the animal model, adiponectin inhibits osteoclastogenesis, reduces bone resorption and increases bone mass (Oshima et al., 2005). At the same time, there is evidence that an increase in adiponectin activates RANKL and inhibits osteoprotegerin, resulting in a decrease in bone formation (Pacheco-Pantoja et al., 2014).

There is no consensus on the effects of glucocorticoids on the expression of adiponectin. Some sources describe that glucocorticoids reduce the serum adiponectin concentration (de Oliveira et al., 2011). While in the studies of Jang et al. (2008) an increase in the serum adiponectin level after therapy with dexamethasone has been observed. One of the main reasons for the contradictory results of glucocorticoids exposure to adiponectin expression is that its expression is regulated by a variety of mechanisms (various transcription factors, signaling cascades and hormones). For example, glucocorticoids increase the concentration of glucose in the blood, which subsequently increases the concentration of insulin, which can regulate the expression of adiponectin. Glucocorticoids affect adipogenesis and differentiation of adipocytes, as well as adipogenic transcription factors that can influence the expression of adiponectin. In addition, glucocorticoids are powerful anti-inflammatory agents and cause a decrease in pro-inflammatory cytokines, which can also indirectly regulate the expression of adiponectin. The observed decrease in the level of adiponectin in the serum of animals of the group with the violation of bone tissue remodeling with the help of glucocorticoids is associated with the direct influence of glucocorticoids on the overall energy metabolism, which complexly regulates the processes of bone remodeling, as well as the fact that the concentrations of adiponectin in the blood do not satisfactorily reflect its products by adipose tissue. An excess of glucocorticoids reduces the level of adiponectin and its activating effect on osteoblasts, reinforcing the processes of resorption in the balance of bone remodeling.

An important role in bone tissue remodeling is played by visfatin, which is a $52 \mathrm{kDa}$ adipocytokine hormone secreted primarily by visceral fat. Visfatin is a mediator of inflammation and acts as a proinflammatory cytokine (Laiguillon et al., 2014), stimulating the production of interkeukin-1 $\beta$, interkeukin-6, tumor necrosis factor- $\alpha$, which confirms the correlation between the content of visfatin and proinflammatory interleukin-17. Visfatin has insulin-mimicking properties and has a hypoglycemic effect (Cinar \& Gurlek, 2013). The role of visfatin in endothelial dysfunction has not been adequately studied, but it has been found that visfatin enhances the expression of adhesion molecules by activating the NF-kB signaling pathway (Lee et al., 2009). These data are confirmed by the positive correlation we found between the levels of visfatin and P-selectin.

Studies have shown that the synthesis and secretion of visfatin are modulated by glucocorticoids, tumor necrosis factor- $\alpha$, interkeukin- 6 and growth factors (Al-Suhaimi \& Shehzad, 2013). There is evidence that visfatin may contribute to bone destruction with an increase in adipogenic differentiation in osteoporosis (Tsiklauri et al., 2016). On the other hand, this adipokine can stimulate the proliferation of osteoblasts and be a negative regulator of osteoclastogenesis, suppressing the differrentiation of monocytes into osteoclasts (Liu et al., 2013). The reason for such discrepancies in the published data related to visfatin remains to be determined. The decrease in the level of visfatin in our experiment, apparently, is due to the depletion of the compensatory reserves of the bone tissue remodeling regulation system, caused by the long-term intake of glucocorticoids into the body.

Studies show that adiponectin and visfatin are oppositely related to the mineral density of bone tissue. Although the mechanisms underlying these correlations are unclear, modulation of bone metabolism by these adipokines can be proposed (Iacobellis et al., 2011). In our research the opposite situation is observed. In the study of intragroup relationships in the control group, direct correlations of average strength between the level of adiponectin, visfatin, and bone density were found
( $r=0.404$ and $r=0.628$, respectively, $\mathrm{P}>0.05$ ), but these relationships were not significant. In the group with experimental osteoporosis, the correlation between the content of adiponectin and bone density changed direction ( $r=-0.214, P>0.05$ ), while the relationship between the content of visfatin and bone density remained straight $(r=0.380, P>$ 0.05 ). The data obtained may indicate the complexity and ambiguity of the role of adiponectin and visfatin in the regulation of bone metabolism. Thus, the use of glucocorticoids leads to disruption in the regulation of bone remodeling, reflected by hormones and intercellular mediators. The revealed correlations are an important indicator of changes in the system of regulation of bone tissue remodeling. The appearance of new connections and a change in their direction between pairs of intercellular mediators can be a manifestation of one of the mechanisms of bone tissue remodeling under the influence of glucocorticoids.

\section{Conclusions}

Reduction of bone mineral density and histological analysis of bone samples of a group of animals with a violation of bone tissue remodeling by glucocorticoids show that the use of dexamethasone has made it possible to obtain a model of bone remodeling disturbance. The studied changes in the concentrations of hormones and intercellular mediators indicate their important role in disturbances in the regulation of bone tissue remodeling under the influence of glucocorticoids. The imbalance between the RANKL and OPG levels, which results from the violation of the feedback mechanism, promotes bone resorption and, consequently, leads to a disturbance of bone remodeling. The degree of participation of adiponectin and visfatin in regulatory interactions of bone and energy metabolism was determined taking into account the interrelation of these adipokines. Correlations found during the study reflect the relationship in the system of regulation of bone tissue remodeling under the influence of glucocorticoids. A complex system of regulating bone remodeling, which includes many factors and their interactions, requires further study, which in the future can lead to the development of methods for treating patients with osteoporosis.

\section{References}

Adamopoulos, I. E., Chao, C., Geissler, R., Laface, D., Blumenschein, W., Iwakura, Y., McClanahan, T., \& Bowman, E. P. (2010). Interleukin-17A upregulates receptor activator of NF- $\mathrm{KB}$ on osteoclast precursors. Arthritis Research and Therapy, 12(1), R29.

Al-Suhaimi, E. A., \& Shehzad, A. (2013). Leptin, resistin and visfatin: The missing link between endocrine metabolic disorders and immunity. European Journal of Medical Research, 18(1), 12.

Avtandilov, G. G. (1990). Medicinskaja morfometrija [Medical morphometry]. Medicina, Moscow (in Russian).

Brennan-Speranza, T. C., Henneicke, H., Gasparini, S. J., Blankenstein, K. I., Heinevetter, U., Cogger, V. C., Svistounov, D., Zhang, Y., Cooney, G. J., Buttgereit, F., Dunstan, C. R., Gundberg, C., Zhou, H., \& Seibel, M. J. (2012). Osteoblasts mediate the adverse effects of glucocorticoids on fuel metabolism. Journal of Clinical Investigation, 122(11), 4172-4189.

Canalis, E., Mazziotti, G., Giustina, A., \& Bilezikian, J. P. (2007). Glucocorticoidinduced osteoporosis: Pathophysiology and therapy. Osteoporosis International, 18(10), 1319-1328.

Cinar, N., \& Gurlek, A. (2013). Association between novel adipocytokines adiponectin, vaspin, visfatin, and thyroid: An experimental and clinical update. Endocr Connect, 2(4), R30-R38.

Crane, J. L., Xian, L., \& Cao, X. (2016). Role of TGF- $\beta$ signaling in coupling bone remodeling. Methods in Molecular Biology, 1344, 287-300.

Davey, R. A., \& Findlay, D. M. (2013). Calcitonin: Physiology or fantasy? Journal of Bone and Mineral Research, 28(5), 973-979.

de Oliveira, C., Iwanaga-Carvalho, C., Mota, J. F., Oyama, L. M., Ribeiro, E. B., \& Oller do Nascimento, C. M. (2011). Effects of adrenal hormones on the expression of adiponectin and adiponectin receptors in adipose tissue, muscle and liver. Steroids, 76(12), 1260-1267.

Florencio-Silva, R., Sasso, G. R., Sasso-Cerri, E., Simões, M. J., \& Cerri, P. S. (2015). Biology of bone tissue: Structure, function, and factors that influence bone cells. BioMed Research International, 2015, 421746.

Iacobellis, G., Iorio, M., Napoli, N., Cotesta, D., Zinnamosca, L., Marinelli, C., Petramala, L., Minisola, S., D'Erasmo, E., \& Letizia, C. (2011). Relation of adiponectin, visfatin and bone mineral density in patients with metabolic syndrome. Journal of Endocrinological Investigation, 34(1), 12-15. 
Jang, C., Inder, W. J., Obeyesekere, V. R., \& Alford, F. P. (2008). Adiponectin, skeletal muscle adiponectin receptor expression and insulin resistance following dexamethasone. Clinical Endocrinology, 69(5), 745-750.

Kaneko, K., Kusunoki, N., Hasunuma, T., \& Kawai, S. (2012). Changes of serum soluble receptor activator for nuclear factor- $\kappa b$ ligand after glucocorticoid therapy reflect regulation of its expression by osteoblasts. Journal of Clinical Endocrinology and Metabolism, 97(10), 1909-1917.

Klimova, Z. A., Zaft, A. A., \& Zaft, V. B. (2014). Sovremennaja laboratornaja diagnostika osteoporoza [Modern laboratory diagnosis of osteoporosis]. International Journal of Endocrinology, 63(7), 75-84 (in Russian).

Laiguillon, M. C., Houard, X., Bougault, C., Gosset, M., Nourissat, G., Sautet, A., Jacques, C., Berenbaum, F., \& Sellam, J. (2014). Expression and function of visfatin (Nampt), an adipokine-enzyme involved in inflammatory pathways of osteoarthritis. Arthritis Research and Therapy, 16(1), R38.

Lee, W. J., Wu, C. S., Lin, H., Lee, I. T., Wu, C. M., Tseng, J. J., Chou, M. M., \& Sheu, W. H. (2009). Visfatin-induced expression of inflammatory mediators in human endothelial cells through the NF- $\mathrm{BB}$ pathway. International Journal of Obesity (London), 33(4), 465-472.

Li, G. W., Xu, Z., Chen, Q. W., Chang, S. X., Tian, Y. N., \& Fan, J. Z. (2013). The temporal characterization of marrow lipids and adipocytes in a rabbit model of glucocorticoid-induced osteoporosis. Skeletal Radiology, 42(9), 1235-1244.

Liu, Y., Chen, Y., Zhao, H., Zhong, L., Wu, L., \& Cui, L. (2011). Effects of different doses of dexamethasone on bone qualities in rats. Sheng Wu Yi Xue Gong Cheng Xue Za Zhi, 28(4), 737-743 (in Chinese).

Liu, Y., Song, C. Y., Wu, S. S., Liang, Q. H., Yuan, L. Q., \& Liao, E. Y. (2013). Novel adipokines and bone metabolism. International Journal of Endocrinology, 2013, 895045.

Lu, X., McCoy, K. S., Hu, W., Xu, J., Wang, H., Chen, P., \& Chen, H. (2013). Dexamethasone reduces IL-17 and Tim-3 expression in BALF of asthmatic mice. Joumal of Huazhong University of Science and Technology. Medical Sciences, 33(4), 479-484.

Luan, X., Lu, Q., Jiang, Y., Zhang, S., Wang, Q., Yuan, H., Zhao, W., Wang, J., \& Wang, X. (2012). Crystal structure of human rankl complexed with its decoy receptor osteoprotegerin. Journal of Immunology, 189(1), 245-252.

Mazziotti, G., Formenti, A. M., Adler, R. A., Bilezikian, J. P., Grossman, A., Sbardella, E., Minisola, S., \& Giustina, A. (2016). Glucocorticoid-induced osteoporosis: Pathophysiological role of GH/IGF-I and PTH/VITAMIN D axes, treatment options and guidelines. Endocrine, 54(3), 603-611.

McKinley, L., Alcom, J. F., Peterson, A., Dupont, R. B., Kapadia, S., Logar, A., Henry, A., Irvin, C. G., Piganelli, J. D., Ray, A., \& Kolls, J. K. (2008). TH17 cells mediate steroid-resistant airway inflammation and airway hyperresponsiveness in mice. Journal of Immunology, 181(6), 4089-4097.

Morita, M., Yoshida, S., Iwasaki, R., Yasui, T., Sato, Y., Kobayashi, T., Watanabe, R., Oike, T., Miyamoto, K., Takami, M., Ozato, K., Deng, C.-X., Aburatani, H., Tanaka, S., Yoshimura, A., Toyama, Y., Matsumoto, M., Nakamura, M., Kawana, H., Nakagawa, T., \& Miyamoto, T. (2016). Smad4 is required to inhibit osteoclastogenesis and maintain bone mass. Scientific Reports, 6, 35221.

Muruganandan, S., \& Sinal, C. J. (2014). The impact of bone marrow adipocytes on osteoblast and osteoclast differentiation. International Union of Biochemistry and Molecular Biology Life, 66, 147-155.

Oshima, K., Nampei, A., Matsuda, M., Iwaki, M., Fukuhara, A., Hashimoto, J., Yoshikawa, H., \& Shimomura, I. (2005). Adiponectin increases bone mass by suppressing osteoclast and activating osteoblast. Biochemical and Biophysical Research Communications, 331(2), 520-526.
Pacheco-Pantoja, E. L., Fraser, W. D., Wilson, P. J., \& Gallagher, J. A. (2014). Differential effects of adiponectin in osteoblast-like cells. Journal of Receptors and Signal Transduction Research, 34(5), 351-360.

Padmalayam, I., \& Suto, M. (2013). Role of adiponectin in the metabolic syndrome: Current perspectives on its modulation as a treatment strategy. Current Pharmaceutical Design, 19(32), 5755-5763.

Plotkin, L. I., Weinstein, R. S., Parfitt, A. M., Roberson, P. K., Manolagas, S. C., \& Bellido, T. (1999). Prevention of osteocyte and osteoblast apoptosis by bisphosphonates and calcitonin. Journal of Clinical Investigation, 104, 1363-1374.

Podkovkin, V. G., Ivanov, D. G., \& Ivanov, G. A. (2008). Vlijanie postojannogo magnitnogo polja na sostojanie kostnoj tkani krys s povyshennym urovnem rezorbcii [The effect of magnetic field on the bone tissue status in rats with high level bone resorption]. Advances in Current Natural Sciences. Biological Sciences, 7, 13-16 (in Russian).

Remuzgo-Martínez, S., Genre, F., López-Mejías, R., Ubilla, B., Mijares, V., Pina, T., Corrales, A., Blanco, R., Martín, J., Llorca, J., \& González-Gay, M. A (2016). Expression of osteoprotegerin and its ligands, RANKL and TRAIL, in rheumatoid arthritis. Scientific reports, 6, 29713.

Sage, A. P., Tintut, J., \& Demer, L. L. (2010). Regulatory mechanisms in vascular calcification. Nature Reviews Cardiology, 7(9), 528-536.

Scialla, J. J., Leonard, M. B., Townsend, R. R., Appel, L., Wolf, M., Budoff, M. J., Chen, J., Lustigova, E., Gadegbeku, C. A., Glenn, M., Hanish, A., Raj, D., Rosas, S. E., Seliger, S. L., Weir, M. R., Parekh, R. S., \& CRIC Study Group. (2011). Correlates of osteoprotegerin and association with aortic pulse wave velocity in patients with chronic kidney disease. Clinical Journal of the American Society of Nephrology, 6(11), 2612-2619.

Sharma, S., Tandon, V. R., Mahajan, S., Mahajan, V., \& Mahajan, A. (2014). Obesity: Friend or foe for osteoporosis. Joumal of Mid-Life Health, 5(1), 6-9.

Tsiklauri, L., Werner, J., Frommer, K. W., Müller-Ladner, U., Rehart, S., Wenisch, S., \& Neumann, E. (2016). Differentiation of spongiosa-derived mesenchymal stromal cells from osteoporosis and osteoarthritis patients are influenced by adipokines. Arthritis and Rheumatology, 68(10).

Tu, Q., Zhang, J., Dong, L. Q., Saunders, E., Luo, E., Tang, J., \& Chen, J. (2011). Adiponectin inhibits osteoclastogenesis and bone resorption via APPL1mediated suppression of Akt1. Joumal of Biological Chemistry, 286(14), 12542-12553.

Wan, M., Li, C., Zhen, G., Jiao, K., He, W., Jia, X., Wang, W., Shi, C., Xing, Q. Chen, Y. F., Jan De Beur, S., Yu, B., \& Cao, X. (2012). Injury-activated TGF $\beta$ controls mobilization of MSCs for tissue remodeling. Stem cells, 30(11), 2498-2511.

Weinstein, R. S. (2011). Clinical practice. Glucocorticoid-induced bone disease. New England Journal of Medicine, 365(1), 62-70.

Xiping, Z., Jun, F., Jie, Z., Bingyan, Y., Jing, M., Wei, Z., Jing, Y., Penghui, J., Wenqin, Y., Ninnin, Z., \& Jiao, H. (2010). Influence of dexamethasone on the expression levels of P-selectin protein in multiple organs of rats with severe acute pancreatitis. Inflammation Research, 59(1), 31-39.

Yamashita, H., Ochiai, H., Saito, A., Shintani, S., \& Azuma, T. (2014). Phosphoinositide 3-kinase (PI3K) activation is differentially regulated during osteogenesis induced by TGF- $\beta 1$ and BMP-2/BMP-7. Journal of Hard Tissue Biology, 23(1), 9-14

Zeng, M., Li, Z.-Y., Ma, J., Cao, P.-P., Wang, H., Cui, Y.-H., \& Liu, Z. (2015). Clarithromycin and dexamethasone show similar anti-inflammatory effects on distinct phenotypic chronic rhinosinusitis: An explant model study. BMC Immunology, 16, 37.

Zubairova, L. D., Mustafin, I. G., \& Nabiullina, R. M. (2013). Patogeneticheskie podhody $\mathrm{k}$ issledovaniju markerov venoznogo tromboza [Pathogenetic approach to venous thrombosis markers examination]. Vestnik of Kazan State Medical University, 94(5), 685-692 (in Russian). 